\title{
ÜBER LOKAL SCHLICHTE FUNKTIONEN, DEREN ERSTE ABLEITUNG BESCHRÄNKTES ARGUMENT HAT
}

\author{
K. DOPPEL \\ Herrn Professor Dr. Rolf Nevanlinna in Verehrung gewidmet
}

1. Einleitung. Bekanntlich kann eine Schwarz-Christoffelsche Funktion $f(z)$, die den Einheitskreis $|z|<1$ auf das Innere eines $n$-Ecks mit den Außenwinkeln $\mu_{n} \pi$ abbildet, vermöge der Sprungfunktion

$$
\psi(\vartheta)=\sum_{0 \leqq \vartheta_{n} \leqq \vartheta} \mu_{n} \pi
$$

als ein Lebesgue-Stieltjes Integral

$$
\log f^{\prime}(z)=-\frac{1}{\pi} \int_{0}^{2 \pi} \log \left(1-e^{-i \vartheta} z\right) d \psi(\vartheta)
$$

geschrieben werden. E. Study hat in seiner Monographie [14] gezeigt, daß die Formel (1) genau dann eine konforme Abbildung $f(z)$ des Einheitskreises $|z|<1$ auf ein konvexes Gebiet vermittelt, wenn $\psi(\vartheta)$ eine auf dem Intervall $0 \leqq \vartheta \leqq 2 \pi$ monotone Funktion darstellt. Die gleichzeitig gestellte Frage, welche konformen Abbildungen des Einheitskreises die Formel (1) erklärt, wenn $\psi(\vartheta)$ eine beliebige Funktion beschränkter Totalvariation ist, blieb zunächst offen.

V. Paatero hat in [9] dieses Problem gelöst und gezeigt, daß die gemäß (1) definierten Funktionen dann dadurch charakterisiert sind, daß der Rand des Bildgebiets von beschränkter Drehung ist. Der dabei eingehende Begriff der Randdrehung eines Gebietes, die beim Vorhandensein einer stetig veränderlichen Randtangente einfach durch die Gesamtvariation des Richtungswinkels der Randtangente und andernfalls durch einen geeigneten Grenzproze $\beta$ zu definieren ist, wurde wohl erstmals von K. Löwner erwähnt [7], ohne freilich auf den Zusammenhang mit der entsprechenden Abbildungsfunktion einzugehen.

Der Beweis von Study stützt sich wesentlich auf die Schwarz-Christoffelsche Formel, indem konvexe Gebiete durch solche mit Polygonen als Randkurven approximiert werden; beim Beweis von Paatero werden aber die konformen Abbildungen des Einheitskreises auf Gebiete mit beschränkter Randdrehung direkt untersucht. 
Zunächst wird bewiesen, daß für jede analytische Funktion $f(z)$, die $|z|<1$ auf ein Gebiet mit beschränkter Randdrehung $\alpha$ abbildet, gilt:

$$
\alpha=\lim _{r \rightarrow 1} \int_{0}^{2 \pi}\left|\operatorname{Re}\left(1+r e^{i t} \frac{f^{\prime \prime}\left(r e^{i t}\right)}{f^{\prime}\left(r e^{i t}\right)}\right)\right| d t .
$$

Anschließend wird die Schwarzsche Formel erweitert und gezeigt, daß nun andererseits die in $|z|<1$ analytische Funktion $1+z f^{\prime \prime}(z) / f^{\prime}(z)$ durch ein Poisson-Stieltjessches Integral

$$
1+z \frac{f^{\prime \prime}(z)}{f^{\prime}(z)}=\frac{1}{2 \pi} \int_{0}^{2 \pi} \frac{e^{i \vartheta}+z}{e^{i \vartheta}-z} d \psi(\vartheta)+i \operatorname{Im} f(0) \quad(|z|<1)
$$

dargestellt werden kann, wo $\psi(\vartheta)$ eine auf dem Intervall $0 \leqq \vartheta \leqq 2 \pi$ reellwertige Funktion mit der Totalvariation $\alpha$ ist. Aus der Formel (3) wird schließlich die Lebesgue-Stieltjessche Integraldarstellung (1) gefolgert. Zur Vollständigkeit sei bemerkt, daß solche Erweiterungen der Schwarzschen Formel von mehreren Autoren gegeben worden sind; vergleiche dazu im Fall von positiv harmonischen Funktionen G. Herglotz [4] und C. Carathéodory [3], von harmonischen Funktionen A. Plessner [11] und im Falle von analytischen Funktionen R. Nevanlinna [8].

Bei den Untersuchungen der Klasse der Funktionen mit beschränkter Randdrehung hat sich die obige Integraldarstellung als nützlich erwiesen; man vergleiche dazu V. Paatero [10], O. Lehto [6], D. A. Brannan [2] und G. Schober. [13].

Nun existieren aber meines Wissens keine Untersuchungen darüber, ob sich die Klasse der durch das obige Lebesgue-Stieltjessche Integral darstellbaren Funktionen erweitern läßt. Dies ist aber naheliegend, falls man von der Tatsache ausgeht, daß zur Existenz des obigen Lebesgue-Stieltjesschen Integrals die beschränkte Totalvariation von $\psi(\vartheta)$ hinreichend aber nicht notwendig ist. Zunächst kann auf Grund des Fatouschen Satzes die Funktion $\psi(\vartheta)$ näher charakterisiert werden. Nach (3) gilt nämlich

$$
u(z)=\operatorname{Re}\left(1+z \frac{f^{\prime \prime}(z)}{f^{\prime}(z)}\right)=\frac{1}{2 \pi} \int_{0}^{2 \pi} \operatorname{Re}\left(\frac{e^{i \vartheta}+z}{e^{i \vartheta}-z}\right) d \psi(\vartheta) .
$$

Da $\psi(\vartheta)$ von beschränkter Totalvariation ist, folgt (vergleiche etwa [12], S. 46), daß die radialen Grenzwerte der Funktion $u(z)$ fast überall existieren und dort mit den Werten der Ableitung $\psi^{\prime}(\vartheta)$ übereinstimmen, d. h. für fast alle $\vartheta, 0 \leqq \vartheta$ $\leqq 2 \pi$, gilt

Wegen

$$
\lim _{r \rightarrow 1} \operatorname{Re}\left(1+r e^{i \vartheta} \frac{f^{\prime \prime}\left(r e^{i \vartheta}\right)}{f^{\prime}\left(r e^{i \vartheta}\right)}\right)=\psi^{\prime}(\vartheta)
$$

$$
\frac{\partial}{\partial \vartheta}\left(\arg f^{\prime}\left(r e^{i \vartheta}\right)\right)=\operatorname{Re}\left(r e^{i \vartheta} \frac{f^{\prime \prime}\left(r e^{i \vartheta}\right)}{f^{\prime}\left(r e^{i \vartheta}\right)}\right)
$$


folgt aber dann auch, daß die radialen Grenzwerte von $\arg f^{\prime}\left(r e^{i \vartheta}\right)$ fast überall existieren. Nach (5) gilt daher bei beliebigem reellem $c$ für fast alle $\vartheta, 0 \leqq \vartheta \leqq 2 \pi$,

$$
\lim _{r \rightarrow 1} \arg f^{\prime}\left(r e^{i \vartheta}\right)=\psi(\vartheta)+\vartheta+c .
$$

Somit besitzt eine analytische Funktion $f(z)$, die den Einheitskreis $|z|<1$ auf ein Gebiet von beschränkter Randdrehung abbildet, die Darstellung

$$
\log f^{\prime}(z)=-\frac{1}{\pi} \int_{0}^{2 \pi} \log \left(1-e^{-i \vartheta} z\right) d\left(\arg f^{\prime}\left(e^{i \vartheta}\right)-\vartheta\right)
$$

mit

$$
\alpha=\int_{0}^{2 \pi}\left|d\left(\arg f^{\prime}\left(e^{i \vartheta}\right)-\vartheta\right)\right|
$$

Ausgehend von diesem Sachverhalt ist nun naheliegend, den Darstellungssatz von Paatero auf die Klasse der im Einheitskreis $|z|<1$ analytischen Funktionen $f(z)$ mit $f^{\prime}(z) \neq 0$ und $\sup \left|\arg f^{\prime}(z)\right|<\infty$ für $|z|<1$ zu verallgemeinern; freilich ist dann die Funktion $\psi(\vartheta)$ nicht mehr von beschränkter Totalvariation, sondern eine passende summierbare Funktion aus $L^{\infty}[0,2 \pi]$. Der im folgenden angegebene Beweis zeigt wieder, daß diese summierbaren Funktionen durch die radialen Grenzwerte von $\arg f^{\prime}\left(r e^{i \vartheta}\right)$ für fast alle $\vartheta$ bestimmt sind. Im Anschluß daran werden mit Hilfe der gefundenen Integraldarstellung für die betrachteten Funktionen einige Eigenschaften aufgezeigt, die auch im Zusammenhang mit Resultaten von H. Hornich [5] stehen.

2. Die Klasse $H_{x}$ und ein Darstellungssatz. Sei $H_{x}$ die Menge der im offenen Einheitskreis $|z|<1$ analytischen Funktionen $f(z)=z+a_{2} z^{2}+\ldots$, die dort lokal schlicht sind $\left(f^{\prime}(z) \neq 0\right)$ und für die dort $\sup \left|\arg f^{\prime}(z)\right| \leqq \pi x$ gilt. Für die Klasse $H_{x}$ gilt folgender Darstellungssatz:

Satz 1. Jede Funktion $f(z)$ aus $H_{x}$ kann mit Hilfe eines Lebesgue-Stieltjesschen Integrals (1) dargestellt werden, d.h. es.gilt

$$
\log f^{\prime}(z)=-\int_{0}^{2 \pi} \log \left(1-e^{-i \vartheta} z\right) d \psi(\vartheta) \quad(|z|<1)
$$

wobei $\psi(\vartheta)$ eine Funktion aus $L^{\infty}[0,2 \pi]$ ist, die für fast alle $\vartheta$ durch die radialen Grenzwerte von $\arg f^{\prime}(z)$ bestimmt ist und für die gilt

$$
\int_{0}^{2 \pi} \psi(\vartheta) d \vartheta=0 \quad \text { und } \quad\|\psi\|_{\infty}=\underset{0 \leqq \vartheta \leqq 2 \pi}{\operatorname{ess} \sup }|\psi(\vartheta)| \leqq \varkappa .
$$

Umgekehrt stellt für eine solche Funktion $\psi(\vartheta)$ das Integral (7) eine Funktion aus $H_{x}$ dar. 
Beweis. Falls $f(z)$ eine Funktion aus $H_{\varkappa}$ ist, so ist $\log f^{\prime}(z)=u(z)+i v(z)$ eine im Einheitskreis $|z|<1$ analytische Funktion mit dort beschränktem Imaginärteil $v(z)$. Für $0 \leqq r<1$ bilden die Funktionen

$$
V_{r}(\vartheta)=\int_{0}^{\vartheta} v\left(r e^{i t}\right) d t
$$

eine gleichgradig absolut stetige Funktionenfamilie. Nach einem Satz von Evans und Bray (vergleiche [12], S. 43) besitzt $v(z)$ eine Poisson-Lebesguesche Integraldarstellung der Gestalt:

$$
v(z)=\frac{1}{2 \pi} \int_{0}^{2 \pi} \operatorname{Re}\left(\frac{e^{i \vartheta}+z}{e^{i \vartheta}-z}\right) \psi^{\sim}(\vartheta) d \vartheta \quad(|z|<1) .
$$

Nach dem Satz von Fatou (vergleiche [12], S. 45) ist $\psi^{\sim}(\vartheta)$ für fast alle $\vartheta$ durch die radialen Grenzwerte von $v(z)$ bestimmt; d. h. für fast alle $\vartheta, 0 \leqq \vartheta \leqq 2 \pi$, gilt

$$
\lim _{r \rightarrow 1} v\left(r e^{i \vartheta}\right)=\lim _{r \rightarrow 1} \arg f^{\prime}\left(r e^{i \vartheta}\right)=\psi^{\sim}(\vartheta) .
$$

Also ist $\psi^{\sim}(\vartheta)$ als Grenzfunktion einer fast überall konvergenten Folge von stetigen Funktionen meßbar und wegen $|v(z)| \leqq \pi \varkappa$ für $|z|<1$ sogar wesentlich beschränkt und stellt somit eine auf dem Intervall $0 \leqq \vartheta \leqq 2 \pi$ summierbare Funktion dar, für die wegen der Voraussetzung $f^{\prime}(0)=1$ nach (9) gilt

Weiter folgt aus (9)

$$
\int_{0}^{2 \pi} \psi^{\sim}(\vartheta) d \vartheta=0
$$

$$
\sup _{|z|<1}|v(z)| \leqq\left\|\psi^{\sim}\right\|_{\infty} \frac{1}{2 \pi} \int_{0}^{2 \pi} \operatorname{Re}\left(\frac{e^{i \vartheta}+z}{e^{i \vartheta}-z}\right) d \vartheta=\left\|\psi^{\tilde{\nu}}\right\|_{\infty}
$$

und daraus wegen (10)

$$
\left\|\psi^{\tilde{\nu}}\right\|_{\infty}=\sup _{|z|<1}|v(z)| \leqq \pi x .
$$

Andererseits stellt das Integral

$$
\frac{i}{2 \pi} \int_{0}^{2 \pi} \frac{e^{i \vartheta}+z}{e^{i \vartheta}-z} \psi^{\sim}(\vartheta) d \vartheta \quad(|z|<1)
$$

eine im offenen Einheitskreis $|z|<1$ analytische Funktion dar, die für $z=0$ verschwindet; weil der Imaginärteil von (11) für $|z|<1$ mit (9) übereinstimmt, folgt für eine beliebige Funktion $f(z)$ aus $H_{x}$

$$
\log f^{\prime}(z)=\frac{i}{2 \pi} \int_{0}^{2 \pi} \frac{e^{i \vartheta}+z}{e^{i \vartheta}-z} \psi^{\sim}(\vartheta) d \vartheta \quad(|z|<1)
$$


und weiter

$$
\frac{f^{\prime \prime}(z)}{f^{\prime}(z)}=\frac{i}{\pi} \int_{0}^{2 \pi} \frac{e^{i \vartheta}}{\left(e^{i \vartheta}-z\right)^{2}} \psi^{\tilde{y}}(\vartheta) d \vartheta=\frac{1}{\pi} \int_{0}^{2 \pi} \psi^{\sim}(\vartheta) d\left(\frac{-1}{e^{i \vartheta}-z}\right) \quad(|z|<1) .
$$

Nach partieller Integration gilt

$$
\frac{f^{\prime \prime}(z)}{f^{\prime}(z)}=\frac{1}{\pi} \int_{0}^{2 \pi} \frac{e^{-i \vartheta}}{1-e^{-i \vartheta} z} d \psi^{\sim}(\vartheta) \quad(|z|<1)
$$

und mit $\psi(\vartheta)=\psi^{\sim}(\vartheta) / \pi$

$$
\frac{d}{d z}\left(\log f^{\prime}(z)\right)=\int_{0}^{2 \pi} \frac{d}{d z}\left[\log \left(1-e^{-i \vartheta} z\right)^{-1}\right] d \psi(\vartheta) \quad(|z|<1) .
$$

Hieraus folgt schließlich die Behauptung (7). Ist umgekehrt $\psi(\vartheta)$ gemäß (8) gegeben, so wird zunächst durch

$$
\log f^{\prime}(z)=-\frac{1}{\pi} \int_{0}^{2 \pi} \log \left(1-e^{-i \vartheta} z\right) d \psi(\vartheta) \quad(|z|<1)
$$

eine in $|z|<1$ analytische Funktion $f(z)$ mit $f^{\prime}(0)=1$ und $f(0)=0$ definiert, die für $|z|<1$ lokal schlicht ist. Weil aus (14) mittels partieller Integration wieder (12) folgt, gilt

$$
v(z)=\operatorname{Im} \log f^{\prime}(z)=\frac{1}{2 \pi} \int_{0}^{2 \pi} \operatorname{Re}\left(\frac{e^{i \vartheta}+z}{e^{i \vartheta}-z}\right) \psi^{\sim}(\vartheta) d \vartheta \quad(|z|<1) .
$$

Daraus ergibt sich nun $\sup |v(z)| \leqq \pi \varkappa$ für $|z|<1$, d. h. die Funktion $f(z)$ liegt in $H_{\varkappa}$, womit der Satz bewiesen ist.

3. Abschätzungen der Funktionen aus $H_{\varkappa}$ und ihrer Ableitungen. Wir beweisen

Satz 2. Für jede Funktion $f(z)$ aus $H_{\varkappa}$ gelten folgende Abschätzungen:

$$
\begin{gathered}
|f(z)| \leqq \sum_{n=0}^{\infty} \frac{1}{n+1} b_{n}|z|^{n+1} \quad(|z|<1), \\
\left(\frac{1-|z|}{1+|z|}\right)^{2 \varkappa} \leqq\left|f^{\prime}(z)\right| \leqq\left(\frac{1+|z|}{1-|z|}\right)^{2 \varkappa} \quad(|z|<1), \\
\left|f^{\prime \prime}(z)\right| \leqq 2 \varkappa \pi\left(\frac{1+|z|}{1-|z|}\right)^{2 \varkappa} \frac{1}{1-|z|^{2}} \quad(|z|<1) .
\end{gathered}
$$

In Abschätzung (15) sind die Zahlen

$$
b_{n}=\sum_{k=0}^{n}\left(\begin{array}{c}
2 \varkappa \\
n-k
\end{array}\right)\left(\begin{array}{c}
2 \varkappa+k-1 \\
k
\end{array}\right)
$$

unabhängig von $f(z)$. 
Beweis. Zunächst erhalten wir aus (7)

(19) $\log \left|f^{\prime}(z)\right|=-\int_{0}^{2 \pi} \log \left|1-e^{i \vartheta} z\right| d \psi(\vartheta)=\int_{0}^{2 \pi} \psi(\vartheta) d \log \left|1-e^{-i \vartheta} z\right| \quad(|z|<1)$.

Wir setzen (bei festem $\left.z=r e^{i t}, 0 \leqq r<1,0 \leqq t<2 \pi\right) \quad h(\vartheta)=\log \left|1-e^{-i \vartheta} z\right|, \quad \vartheta \in \boldsymbol{R}$, und schätzen das Lebesgue-Stieltjessche Integral

$$
\int_{0}^{2 \pi} \psi(\vartheta) d h(\vartheta)
$$

nach oben und unten ab. Indem wir $\psi(\vartheta)$ periodisch auf $\boldsymbol{R}$ fortsetzen, gilt

und

$$
\int_{0}^{2 \pi} \psi(\vartheta) d h(\vartheta)=\int_{t}^{t+2 \pi} \psi(\vartheta) d h(\vartheta)
$$

$$
\frac{d}{d \vartheta}(h(\vartheta)) \begin{cases}>0 & \text { für } 0<\vartheta-t<\pi \\ <0 & \text { für } \pi<\vartheta-t<2 \pi .\end{cases}
$$

Dann ist $h(\vartheta)=h_{1}(\vartheta)-h_{2}(\vartheta)$, wobei die Funktionen

$$
\begin{aligned}
& h_{1}(\vartheta)= \begin{cases}h(\vartheta) & \text { für } t<\vartheta<t+\pi \\
0 & \text { für } t+\pi \leqq \vartheta \leqq t+2 \pi\end{cases} \\
& h_{2}(\vartheta)=\left\{\begin{array}{cc}
0 & \text { für } t \leqq \vartheta \leqq t+\pi \\
-h(\vartheta) & \text { für } t+\pi<\vartheta<t+2 \pi
\end{array}\right.
\end{aligned}
$$

auf $\boldsymbol{R}$ periodisch und im Intervall $t \leqq \vartheta \leqq t+2 \pi$ monoton wachsend sind. Nach (8) ist $\|\psi\|_{\infty} \leqq \varkappa$ und daher gilt

Analog gilt

$$
\begin{aligned}
\int_{t}^{t+2 \pi} \psi(\vartheta) d h_{1}(\vartheta) & \leqq \varkappa \int_{t}^{t+\pi} d h(\vartheta) \\
& =\varkappa\left(\log \left|1+e^{-i t} z\right|-\log \left|1-e^{-i t} z\right|\right) \\
& \leqq \varkappa \log \frac{1+|z|}{1-|z|} .
\end{aligned}
$$

$$
\begin{aligned}
-\int_{t}^{t+2 \pi} \psi(\vartheta) d h_{2}(\vartheta) & \leqq \varkappa \int_{t+\pi}^{t+2 \pi} d h(\vartheta) \\
& =\chi\left(-\log \left|1-e^{-i t} z\right|+\log \left|1+e^{-i t} z\right|\right) \\
& \leqq \chi \log \frac{1+|z|}{1-|z|} .
\end{aligned}
$$

Nach (19)-(21) ergibt sich die Abschätzung nach oben

$$
\log \left|f^{\prime}(z)\right| \leqq 2 \varkappa \log \frac{1+|z|}{1-|z|} \quad(|z|<1)
$$


Auf gleiche Weise schätzt man nach unten $a b$

$$
\begin{aligned}
\log \left|f^{\prime}(z)\right| & \geqq-\varkappa \int_{0}^{2 \pi} d h_{1}(\vartheta)+\varkappa \int_{0}^{2 \pi} d h_{2}(\vartheta) \\
& \geqq 2 \varkappa \log \left|\frac{1-e^{-i t} z}{1+e^{-i t} z}\right| \\
& \geqq 2 \varkappa \log \frac{1-|z|}{1+|z|} .
\end{aligned}
$$

Also gilt die Behauptung (16).

Aus (16) folgert man

$$
|f(z)| \leqq \int_{0}^{|z|}\left|f^{\prime}(z)\right| d|z|=\int_{0}^{|z|}\left(\frac{1+|z|}{1-|z|}\right)^{2 x} d|z| \quad(|z|<1) .
$$

Wennman den Integranden des rechten Integrals nach Potenzen von $|z|$ entwickelt und gliedweise integriert sowie

$$
\left(\frac{1+|z|}{1-|z|}\right)^{\alpha}=\sum_{n=0}^{\infty} b_{n}|z|^{n}
$$

berücksichtigt, ergibt sich Abschätzung (15).

Andererseits folgt aus (13) mit $\psi(\vartheta)=\psi^{\sim}(\vartheta) / \pi$

sowie

$$
f^{\prime \prime}(z)=i f^{\prime}(z) \int_{0}^{2 \pi} \frac{e^{i \vartheta}}{\left(e^{i \vartheta}-z\right)^{2}} \psi(\vartheta) d \vartheta \quad(|z|<1)
$$

$$
\left|f^{\prime \prime}(z)\right| \leqq\left|f^{\prime}(z)\right| \varkappa \int_{0}^{2 \pi} \frac{1}{\left|e^{i \vartheta}-z\right|^{2}} d \vartheta=\left|f^{\prime}(z)\right| \frac{2 \varkappa \pi}{1-|z|^{2}} .
$$

Gemäß (16) gilt schließlich die Behauptung (17).

4. Extremalfunktionen aus $H_{x}$. Es erhebt sich die Frage, für welche Funktionen die obigen Abschätzungen scharf sind. Aus (20) und (21) geht hervor, daß in (22) für ein $z$ mit $\arg z=t$ genau dann das Gleichheitszeichen steht, wenn

$$
\psi(\vartheta)=\left\{\begin{array}{rlrr}
x & \text { für } & t \leqq \vartheta \leqq t+\pi \\
-\chi & \text { für } & t+\pi<\vartheta<t+2 \pi
\end{array}\right.
$$

ist. Setzt man $\psi(\vartheta)$ periodisch auf $\boldsymbol{R}$ fort und in (7) ein, so wird

und wir erhalten

$$
\log f^{\prime}(z)=2 \varkappa \log \frac{1+e^{-i t} z}{1-e^{-i t} z},
$$

$$
f^{\prime}(z)=\left(\frac{1+e^{-i t} z}{1-e^{-i t} z}\right)^{2 x}
$$


Die Integration ergibt die in $H_{x}$ liegende Funktion

$$
f(z)=\sum_{n=0}^{\infty} \frac{1}{n+1} b_{n} e^{-i n t} z^{n+1},
$$

wobei $b_{n}$ die Zahlen (18) sind.

Es ist nun unmittelbar klar, daß die hergeleitete Funktion $f(z)$ und $f^{\prime}(z)$ die in Satz 2 angegebenen Schranken im Punkte $z=|z| e^{i t}$ bzw. $z=|z| e^{-i t}$ erreichen. Für $z=|z| e^{i t}$ gilt

und

$$
|f(z)|=\int_{0}^{|z|}\left(\frac{1+|z|}{1-|z|}\right) d|z|=\sum_{n=0}^{\infty} \frac{1}{n+1} b_{n}|z|^{n+1}
$$

bzw. für $z=|z| e^{-i t}$

$$
\left|f^{\prime}(z)\right|=\left(\frac{1+|z|}{1-|z|}\right)^{2 x}
$$

$$
\left|f^{\prime}(z)\right|=\left(\frac{1-|z|}{1+|z|}\right)^{2 x}
$$

Die Schranke für $f^{\prime \prime}(z)$ in Satz 2 wird von der konstruierten Funktion (24) nicht erreicht.

\section{Literatur}

[1] Aharonov, D., und S. Friedland: On functions of bounded boundary rotation. - Ann. Acad. Sci. Fenn. Ser. A. I. Math. 585, 1974, 1-18.

[2] Brannan, D. A.: On coefficient problems for certain power series. - Proceedings of the Symposium on complex analysis, Canterbury 1973. [Edited by J. Clunie and W. K. Hayman.] London Mathematical Society Lecture Note Series 12 . The University Press, Cambridge, 1974, 17-27.

[3] Carathéodory, C.: Über die Fourierschen Koeffizienten monotoner Funktionen. Sitzungsberichte der Preußischen Akademie der Wissenschaften zu Berlin 1920, 559-573.

[4] Herglotz, G.: Über Potenzreihen mit positivem, reellem Teil im Einheitskreis. - Berichte über die Verhandlungen der Königl. Sächsischen Gesellschaft der Wissenschaften zu Leipzig, Mathematisch-physische Klasse 63, 1911, 501-511.

[5] Hornich, H.: Ein Banachraum analytischer Funktionen im Zusammenhang mit den schlichten Funktionen. - Monatsh. Math. 73, 1969, 36-45.

[6] Lehto, O.: On the distortion of conformal mappings with bounded boundary rotation.

- Ann. Acad. Sci. Fenn. Ser. A. I. Math.-Phys. 124, 1952, 1-14.

[7] LöWNER, K.: Untersuchungen über die Verzerrung bei konformen Abbildungen des Einheitskreises $|z|<1$, die durch Funktionen mit nicht verschwindender Ableitung geliefert werden. - Berichte über die Verhandlungen der Königl. Sächsischen Gesellschaft der Wissenschaften zu Leipzig, Mathematisch-physische Klasse 69, 1917, 89-106.

[8] Nevanlinna, R.: Asymptotische Entwicklungen beschränkter Funktionen und das Stieltjessche Momentenproblem. - Ann. Acad. Sci. Fenn. Ser. A. 18:5, 1922, 1-53.

[9] PaAtero, V.: Über die konforme Abbildung von Gebieten, deren Ränder von beschränkter Drehung sind. - Ann. Acad. Sci. Fenn. Ser. A. 33:9, 1931, 1-78. 
[10] PaAtero, V.: Über Gebiete von beschränkter Randdrehung. - Ann. Acad. Sci. Fenn. Ser. A. 37:9, 1933, 1-20.

[11] Plessner, A.: Zur Theorie der konjugierten trigonometrischen Reihen. - Mitt. Math. Sem. Univ. Gießen 10, 1923, 1-36.

[12] Priwalow, I. I.: Randeigenschaften analytischer Funktionen. - [Deutsche Übersetzung von der zweiten, unter Redaktion von A. I. Markuschewitsch überarbeiteten und ergänzten russischen Auflage.] Hochschulbücher für Mathematik 25. VEB Deutscher Verlag der Wissenschaften, Berlin, 1956.

[13] Schober, G.: Univalent functions - Selected topics. - Lecture Notes in Mathematics 478, Springer-Verlag, Berlin-Heidelberg-New York, 1975.

[14] Study, E.: Vorlesungen über ausgewählte Gegenstände der Geometrie. Zweites Heft. Herausgegeben unter Mitwirkung von W. Blaschke. Konforme Abbildung einfach-zusammenhängender Bereiche. - Druck und Verlag von B. G. Teubner, Leipzig-Berlin, 1913.

Freie Universität Berlin

I. Mathematisches Institut

Hüttenweg 9

D 1000 Berlin 33

Eingegangen am 22. Dezember 1977 